International Mathematical Forum, Vol. 9, 2014, no. 18, 857 - 869

HIKARI Ltd, www.m-hikari.com

http://dx.doi.org/10.12988/imf.2014.4480

\title{
Natural Lifts and Curvatures, Arc-Lengths of the Spherical Indicatries of the Evolute Curve in $E^{3}$
}

\author{
Süleyman Şenyurt \\ Ordu University, Faculty of Arts and Sciences \\ Department of Mathematics, 52200 Ordu, Turkey \\ Selma Demet \\ Ordu University, Natural Science Institute \\ Department of Mathematics, 52200 Ordu, Turkey
}

Copyright (C) 2014 Süleyman Şenyurt and Selma Demet. This is an open access article distributed under the Creative Commons Attribution License, which permits unrestricted use, distribution, and reproduction in any medium, provided the original work is properly cited.

\begin{abstract}
In this paper, we investigated the natural lifts and curvatures of the spherical indicatries of the evolute in $E^{3}$. Firstly, it is shown that the Darboux vektor of evolute curve and binormal vektor of the evolute curve are linearly dependent, secondly the relations among the geodesic curvatures and arc-lengths with respect to $E^{3}$ and $S^{2}$ of the fixed pole curve $\left(C^{*}\right)$ and the spherical indicartix curves $\left(T^{*}\right),\left(N^{*}\right),\left(B^{*}\right)$ generated by the unit vektor $\left(C^{*}\right)$ on $S^{2}$ have been obtained and finally, the condition being the natural lifts of the spherical indicatrix of the evolute curve $\left(\alpha^{*}\right)$ are an integral curve of geodesic spray has expressed.
\end{abstract}

Mathematics Subject Classification: 53A04

Keywords: Evolute curve, Natural lift of spherical indicatrix curve, Geodesic spray, Geodesic curvatures

\section{Introduction and Preliminary notes}

We now review some basic concepts on classical differantial geometry of space curves and surfaces in three dimensional Euclidean space $E^{3}$.For any two vec- 
tors $X=\left\{x_{1}, x_{2}, x_{3}\right\}$ and $Y=\left\{y_{1}, y_{2}, y_{3}\right\}$, we denote $\langle X, Y\rangle=\sum x_{i} y_{i}$ as standart inner product. Let $\alpha: I \subset I R \longrightarrow E^{3}, \alpha(s)=\left(\alpha_{1}(s), \alpha_{2}(s), \alpha_{3}(s)\right)$ be a curve with $\dot{\alpha}(s) \neq \overrightarrow{0}$. We also denote the norm of $X$ by $\|X\|$. The arc-length of a curve a measured from $\alpha\left(s_{0}\right), s_{0} \in I$ is

$$
s=\int_{s_{0}}^{s}\|\dot{\alpha}(s)\| d u
$$

We say that a curve $\alpha$ is parametrical by the arc-length if it satisfies $\left\|\alpha^{\prime}(s)\right\|=1$. Throughout this paper we denote the arc-length of space curves. Let us denote $T(s)=\alpha^{\prime}(s)$ and we call a unit tangent vector of $\alpha$ at $s$. We define curvature of $\alpha$ by $\kappa(s)=\left\|\alpha^{\prime \prime}(s)\right\|$. If $\kappa(s) \neq 0$, then the unit principal normal vector $N(s)$ of the curve $\alpha$ by $\alpha^{\prime \prime}(s)=\kappa(s) N(s)$. The unit vector $B(s)=T(s) \times N(s)$ is called a unit binormal vector of the curve $\alpha$ at $s$. Then the set $\{T(s), N(s), B(s)\}$ is called the Frenet frame of the curve $\alpha$ at the point $\alpha(s)$ Then the following Serret-Frenet formula holds:

$$
\begin{aligned}
T^{\prime}(s) & =\kappa(s) N(s) \\
N^{\prime}(s) & =-\kappa(s) T(s)+\tau(s) B(s) \\
B^{\prime}(s) & =-\tau(s) N(s)
\end{aligned}
$$

where $\tau(s)$ is the torsion of the curve $\alpha$.For any unit speed curve $\alpha: I \rightarrow E^{3}$, the vector $W$ is called Darboux vector defined by

$$
W=\tau(s) T(s)+\kappa(s) B(s)
$$

If we consider the normalization of the Darboux $C=\frac{1}{\|W\|} W$, we have

$$
\cos \phi=\frac{\kappa(s)}{\|W\|} \quad \sin \phi=\frac{\tau(s)}{\|W\|}
$$

and

$$
C=\sin \phi T(s)+\cos \phi B(s)
$$

where $\measuredangle(W, B)=\phi$.

$$
\kappa(t)=\frac{\left\|\alpha^{\prime}(t) \times \alpha^{\prime \prime}(t)\right\|}{\left\|\alpha^{\prime}(t)\right\|^{3}}, \quad \tau(t)=\frac{\operatorname{det}\left(\alpha^{\prime}(t), \alpha^{\prime \prime}(t), \alpha^{\prime \prime \prime}(t)\right)}{\left\|\alpha^{\prime}(t) \times \alpha^{\prime \prime}(t)\right\|^{2}}
$$

Furtermore, a curve $\alpha: I \rightarrow E^{3}$ with $\kappa(s) \neq 0$ is called a cylindrical helix if the tangent line of $\alpha$ makes a constant angle with a fixed direction. It has been 
known that the curve $\alpha(s)$ is a cylindrical helix if and only if $\frac{\tau(s)}{\kappa(s)}=$ constant. If both of $\kappa(s) \neq 0$ and $\tau(s)$ are constant, it is, of course,cylindrical helix. We call it a circular helix. Let $M$ be a surface in $E^{3}$ and the curve $\alpha: I \rightarrow M$ be an unit speed.Then, the curve $\alpha: I \rightarrow M$ is called an integral curve of the vector field $X$ if

$$
\frac{d \alpha(s)}{d s}=X(\alpha(s))
$$

The curve $\bar{\alpha}: I \rightarrow T M, \bar{\alpha}(s)=\left(\alpha(s), \alpha^{\prime}(s)\right)$ is called the natural lift of the curve $\alpha: I \rightarrow E^{3}$, where $T M=\cup_{P \in M} T_{M} P$ [1] Then, for all $X \in T M$ the map $S: T M \rightarrow T M$ is called Weingarten map (or shape operator) defined by

$$
S(X)=D_{X} N
$$

where $\mathrm{D}$ is affine connection on $M$. Accordingly, for all $V \in T M$ the vector field $X$ is called geodesic spray on $T M$ defined by [1],

$$
D_{X} V=-\langle V, S(V)\rangle N_{P}, P \in M
$$

Then, the equation as know Gauss equation is

$$
\bar{D}_{X} Y=D_{X} Y+\langle S(X), Y\rangle N
$$

where $N \in T M^{\perp}$. Here $\bar{D}$ become a covariant derivative operator sense of Gauss of a Riemannian connection on $M$. Let $T$ be the unit tangent vector of the curve $\alpha$ on $M$. The curve $\alpha$ is called a geodesic curve in $E^{3}$ satisfied by

$$
D_{T} T=0 \text {. }
$$

Similarly, the curve $\alpha$ is called a geodesic curve in $M$ satisfied by

$$
\bar{D}_{T} T=0
$$

Then, we know that the geodesic curvature of t9he curve $\alpha$ on $M$ defined by

$$
k_{g}=\left\|\bar{D}_{T} T\right\|
$$

where $T$ is the unit tangent vector of the curve $\alpha$ on $M$. The geodesic curvature of the curve $\alpha$ on $M$ at $P \in M$ is the vector projection of the curvature vector $k$ of $\alpha$ at $P$ onto the tangent plane $T_{M}(P)$.Similarly, the geodesic curvature of the curve $\alpha$ in $E^{3}$ defined by

$$
\gamma_{g}=\left\|D_{T} T\right\|
$$

Let us consider the Frenet frame $\{T, N, B\}$ and the vector $C$. Accordingly, the arc-lengths and the geodesic curvatures of the spherical indicatrix curves 
$(T),(N)$ and $(B)$ with the fixed pole curve $(C)$ with respect to $E^{3}$, respectively, generated by the vectors $T, N$ and $B$ with the vector $C$ on $S^{2}$ are as follows:

$$
\begin{aligned}
& \left\{\begin{array}{l}
s_{T}=\int_{0}^{s} \kappa d s \\
s_{N}=\int_{0}^{s}\|W\| d s \\
s_{B}=\int_{0}^{s} \tau d s \\
s_{C}=\int_{0}^{s} \phi^{\prime} d s \\
k_{T}=\int_{0}^{s} \frac{1}{\cos \phi} d s \\
k_{N}=\int_{0}^{s}\left[1+\left(\frac{\phi^{\prime}}{\|W\|}\right)^{2}\right]^{\frac{1}{2}} d s \\
k_{B}=\int_{0}^{s} \frac{1}{\sin \phi} d s \\
k_{C}=\int_{0}^{s}\left[1+\left(\frac{\|W\|}{\phi^{\prime}}\right)^{2}\right]^{\frac{1}{2}} d s
\end{array}\right.
\end{aligned}
$$

Similarly, the geodesic curvatures of the geodesic curvatures of the spherical indicatrix curves $(T),(N)$ and $(B)$ with the fixed pole curve $(C)$ with respect to $S^{2}$, respectively, generated by the vectors $T, N$ and $B$ with the vector $C$ on $S^{2}$ are as follows:

$$
\left\{\begin{aligned}
\gamma_{T} & =\int_{0}^{s} \tan \phi d s \\
\gamma_{N} & =\int_{0}^{s} \frac{\phi^{\prime}}{\|W\|} d s \\
\gamma_{B} & =\int_{0}^{s} \cot \phi d s \\
\gamma_{C} & =\int_{0}^{s} \frac{\|W\|}{\phi^{\prime}} d s
\end{aligned}\right.
$$

[4] thus we can give the following theorem:

Theorem 1.1 Let $M$ be a surface in $E^{3}$ and the curve $\alpha: I \rightarrow M$ be a unit speed. The natural lift $\bar{\alpha}: I \rightarrow T M$ is an integral curve of the geodesic spray $X$ if and only if the natural lift $\bar{\alpha}$ is a geodesic on $M$.

Result of this theorem, the following corollaries can be given. 
Corollary 1.2 If the curve $\alpha$ is a unit circle, then the tangents indicatrix of the curve $\alpha$ is a great circle on the sphere $S^{2}$. Then, the natural lift $(\bar{T})$ is an integral curve of the geodesic spray on the tangent bundle $T\left(S^{2}\right)$.

Corollary 1.3 If the curve $\alpha$ is a circular helix, then the principal normals indicatrix of the curve $\alpha$ is a great circle on the sphere $S^{2}$. Then, the natural lift $(\bar{N})$ is an integral curve of the geodesic spray on the tangent bundle $T\left(S^{2}\right)$.

Corollary 1.4 There is not exist any curve such that the binormals indicatrix $(B)$ is a great circle on the sphere $S^{2}$. Then, the natural lift $(\bar{B})$ is not an integral curve of the geodesic spray on the tangent bundle $T\left(S^{2}\right)$.

Corollary 1.5 If the curve $\alpha$ is a helix, then the fixed pole curve $(C)$ is a great circle on the sphere $S^{2}$. Then, the natural lift $(\bar{C})$ is an integral curve of the geodesic spray on the tangent bundle $T\left(S^{2}\right)$.

Definition 1.6 Let $\alpha: I \rightarrow E^{3}$ and $\alpha^{*}: I \rightarrow E^{3}$ be the $C^{2}$-class differantiable unit speed two curves of class $C^{2}$ and let $\{T(s), N(s), B(s)\}$ and $\left\{T^{*}(s), N^{*}(s), B^{*}(s)\right\}$ be the Frenet frames of the curves $\alpha$ and $\alpha^{*}$, respectively. For $\forall s \in I$, if tangent of curve $\alpha^{*}$ at point of $\alpha^{*}(s)$ pass through point of $\alpha(s)$ and if $\left\langle T^{*}(s), T(s)\right\rangle=0$, curve $\alpha^{*}$ is called a evolute of curve $\alpha$.

The relations between the Frenet frames $\{T, N, B\}$ and $\left\{T^{*}, N^{*}, B^{*}\right\}$ are as follows:

$$
\left\{\begin{array}{l}
T^{*}=\cos (\varphi+c) N-\sin (\varphi+c) B \\
N^{*}=-T \\
B^{*}=\sin (\varphi+c) N+\cos (\varphi+c) B
\end{array}\right.
$$

Additionally, the relation between curvatures and the torsions are:

$$
\left\{\begin{array}{l}
\kappa^{*}=\frac{\kappa^{3} \cos ^{3}(\varphi+c)}{\kappa \tau \sin (\varphi+c)-\kappa^{\prime} \cos (\varphi+c)} \\
\tau^{*}=\frac{-\kappa^{3} \sin (\varphi+c) \cos ^{2}(\varphi+c)}{\kappa \tau \sin (\varphi+c)-\kappa^{\prime} \cos (\varphi+c)}
\end{array}\right.
$$

where $\measuredangle\left(\alpha^{*}(s)-\alpha(s), N(s)\right)=\varphi(s)+c$ and for $c \in I R$

$$
\varphi(s)=\int_{0}^{s} \tau(u) d u
$$

and

$$
\alpha^{*}(s)=\alpha(s)+\rho(s) N(s)-\rho(s) \tan [\varphi(s)+c] B(s) \quad[3],[6] \text {. }
$$




\section{Natural Lifts and Curvatures of the Spher- ical Indicatrices of the Evolute Curve}

Theorem 2.1 Let $\alpha^{*}$ be evolute of curve $\alpha$.There are following relation between $B$ binormal vector of curve $\alpha$ at point $\alpha(s)$ and $W^{*}$ Darboux vector of curve $\alpha^{*}$ at point $\alpha^{*}(s)$.

$$
W^{*}=\frac{\kappa^{3} \cos (\varphi+c)}{\kappa \tau \sin (\varphi+c)-\kappa^{\prime} \cos (\varphi+c)} B
$$

Proof 2.2 Substituting (8) and (9) into $W^{*}=\tau^{*} T+\kappa^{*} B^{*}$ we get

$$
W^{*}=\frac{\kappa^{3} \cos (\varphi+c)}{\kappa \tau \sin (\varphi+c)-\kappa^{\prime} \cos (\varphi+c)} B
$$

Corollary 2.3 Let $\alpha^{*}$ be evolute of curve $\alpha$. The unit vector $C^{*}$ in direction of the Darboux vector $W$ of curve $\alpha^{*}$ and binormal vector $B$ of curve $\alpha$ are linearly dependent on.

$$
C^{*}=B
$$

Proof 2.4 Substituting (8) and (9) into $C^{*}=\sin \phi^{*} T^{*}+\cos \phi^{*} B^{*}$ and rearranging we get

$$
C^{*}=B
$$

Theorem 2.5 Let $\alpha^{*}$ be evolute of curve $\alpha$. The angle in between Darboux vector $W^{*}$ with binormal vector $B^{*}$ of curve $\alpha^{*}$ is $\phi^{*}$ and there is following connection with each other.

$$
\left\{\begin{array}{l}
\sin \phi^{*}=-\sin (\varphi+c) \\
\cos \phi^{*}=\cos (\varphi+c)
\end{array}\right.
$$

Proof 2.6 Writing numerical values at (9) into $\sin \phi^{*}=\frac{\tau^{*}}{\left\|W^{*}\right\|}$ and $\cos \phi^{*}=$ $\frac{\kappa^{*}}{\left\|W^{*}\right\|}$, with calculation we obtain

$$
\begin{aligned}
& \sin \phi^{*}=-\sin (\varphi+c) \\
& \cos \phi^{*}=\cos (\varphi+c)
\end{aligned}
$$


Now, let compute the arc-lengths with the geodesic curvatures of spherical indicatrix curves with the $\left(T^{*}\right),\left(N^{*}\right)$ and $\left(B^{*}\right)$ with the fixed pole curve $\left(C^{*}\right)$ with the respect to $E^{3}$ and $S^{2}$ : Primarly, by the (5), for the arc-length $s_{T^{*}}$ of tangents indicatrix $\left(T^{*}\right)$ of the evolute curve $\alpha^{*}$ we can write

$$
\begin{gathered}
s_{T^{*}}=\int_{0}^{s}\left\|\frac{d T^{*}}{d s}\right\| d s . \\
T^{*}=\cos (\varphi+c) N-\sin (\varphi+c) B
\end{gathered}
$$

differentiating we get

$$
\frac{d T^{*}}{d s}=-\kappa \cos (\varphi+c) T+\left(\tau-\varphi^{\prime}\right) \sin (\varphi+c) N+\left(\tau-\varphi^{\prime}\right) \cos (\varphi+c) B
$$

$\varphi^{\prime}=\tau$ and normalizing we obtain

$$
s_{T^{*}}=\int_{0}^{s} \kappa \cos (\varphi+c) d s .
$$

For the arc-length $s_{N^{*}}$ of principal normals indicatrix $\left(N^{*}\right)$

$$
s_{N^{*}}=\int_{0}^{s}\left\|\frac{d N^{*}}{d s}\right\| d s .
$$

Compiting, we get

$$
s_{N^{*}}=\int_{0}^{s} \kappa d s
$$

Similarly, for the arc-length $s_{B^{*}}$ of binormals indicatrix $\left(B^{*}\right)$ of the evolute curve $\alpha^{*}$ we can write

$$
\begin{gathered}
s_{B^{*}}=\int_{0}^{s}\left\|\frac{d B^{*}}{d s}\right\| d s . \\
s_{B^{*}}=\int_{0}^{s} \kappa \sin (\varphi+c) d s .
\end{gathered}
$$

Finally, for the arc-length $s_{C^{*}}$ of the fixed pole curve $\left(C^{*}\right)$ we can write

$$
\begin{gathered}
s_{C^{*}}=\int_{0}^{s}\left\|\frac{d C^{*}}{d s}\right\| d s . \\
s_{C^{*}}=\int_{0}^{s} \tau d s
\end{gathered}
$$


Corollary 2.7 Let $\alpha^{*}$ be evolute of curve $\alpha$ in $E^{3}$ and $\left\{T^{*}, N^{*}, B^{*}\right\}$ be Frenet-frame of the evolute curve $\alpha^{*}$ at $\alpha^{*}(s)$. For the arc-lengths of the spherical indicatrix curves $\left(T^{*}\right),\left(N^{*}\right)$ and $\left(B^{*}\right)$ with the fixed pole curve $\left(C^{*}\right)$ with the respect to $E^{3}$ we have

$$
\begin{aligned}
& \text { 1) } s_{T^{*}}=\int_{0}^{s} \kappa \cos (\varphi+c) d s \\
& \text { 2) } s_{N^{*}}=\int_{0}^{s} \kappa d s \\
& \text { 3) } s_{B^{*}}=\int_{0}^{s} \kappa \sin (\varphi+c) d s \\
& \text { 4) } s_{C^{*}}=\int_{0}^{s} \tau d s
\end{aligned}
$$

Now let us compute the geodesic curvatures of spherical indicatrix curves $\left(T^{*}\right),\left(N^{*}\right)$ and $\left(B^{*}\right)$ with the fixed pole curve $\left(C^{*}\right)$ with the respect to $E^{3}$ :

For the geodesic curvature $k_{T^{*}}$ of the tangents indicatrix curve $\left(T^{*}\right)$ of the evolute curve $\alpha^{*}$ we can write

$$
k_{T^{*}}=\left\|D_{T_{T^{*}}} T_{T^{*}}\right\|
$$

Differentiating the curve $\alpha_{T^{*}}\left(s_{T^{*}}\right)=T^{*}\left(s^{*}\right)$ with the respect to $s_{T^{*}}$ and normalizing we obtain

$$
T_{T^{*}}=-T
$$

Computing the vector $D_{T_{T^{*}}} T_{T^{*}}$ we get

$$
\begin{gathered}
D_{T_{T^{*}}} T_{T^{*}}=-\kappa N \cdot \frac{1}{\kappa \cos (\varphi+c)}=-\sec (\varphi+c) N \\
k_{T^{*}}=\sec (\varphi+c)
\end{gathered}
$$

Similarly, we have

$$
\begin{gathered}
k_{N^{*}}=\frac{\|W\|}{\kappa} \\
k_{B^{*}}=\operatorname{cosec}(\varphi+c) \\
k_{C^{*}}=\frac{\|W\|}{\tau} .
\end{gathered}
$$

Then the following corollary can be given. 
Corollary 2.8 Let $\alpha^{*}$ be evolute of curve $\alpha$ in $E^{3}$ and $\left\{T^{*}, N^{*}, B^{*}\right\}$ be Frenet-frame of the evolute curve $\alpha^{*}$ at $\alpha^{*}(s)$. For the geodesic curvatures of the spherical indicatrix curves $\left(T^{*}\right),\left(N^{*}\right)$ and $\left(B^{*}\right)$ with the fixed pole curve $\left(C^{*}\right)$ with the respect to $E^{3}$ we have

$$
\begin{aligned}
& \text { 1) } k_{T^{*}}=\sec (\varphi+c) \\
& \text { 2) } k_{N^{*}}=\frac{\|W\|}{\kappa} \\
& \text { 3) } k_{B^{*}}=\operatorname{cosec}(\varphi+c) \\
& \text { 4) } k_{C^{*}}=\frac{\|W\|}{\tau} .
\end{aligned}
$$

Now let us compute the geodesic curvatures of the spherical indicatrix curves $\left(T^{*}\right),\left(N^{*}\right)$ and $\left(B^{*}\right)$ with the fixed pole curve $\left(C^{*}\right)$ with the respect to $S^{2}$ :

For the geodesic curvature $\gamma_{T^{*}}$ of the tangents indicatrix curve $\left(T^{*}\right)$ of the evolute curve $\alpha^{*}$ we can write

$$
\gamma_{T^{*}}=\left\|\bar{D}_{T_{T^{*}}} T_{T^{*}}\right\|
$$

By (4), (8) and (17) we obtain

$$
\bar{D}_{T_{T^{*}}} T_{T^{*}}=\left(\frac{\sin ^{2}(\varphi+c)}{\cos (\varphi+c)}\right) N-\sin (\varphi+c) B
$$

Normalizing

$$
\gamma_{T^{*}}=\tan (\varphi+c)
$$

If the curve $\left(\overline{T^{*}}\right)$ is an integral curve of the geodesic spray then $\bar{D}_{T_{T^{*}}} T_{T^{*}}=$ 0 . Thus $\sin (\varphi+c)=0$. So, we can give the following corollary.

Corollary 2.9 If the tangent vector of evolute curve $\alpha^{*}$ is equal principal normal of curve $\alpha$, then the natural lift $\left(\overline{T^{*}}\right)$ of the tangent indicatrix $\left(T^{*}\right)$ is an integral curves of the geodesic spray on tangent bundle $T\left(S^{2}\right)$.

For the geodesic curvature $\gamma_{N^{*}}$ of the principal normals indicatrix curve $\left(N^{*}\right)$ of the evolute curve $\alpha^{*}$ with respect to $S^{2}$ we can write

$$
\gamma_{N^{*}}=\left\|\bar{D}_{T_{N^{*}}} T_{N^{*}}\right\|
$$


By direct calculation, the vector $\bar{D}_{T_{N^{*}}} T_{N^{*}}$ is obtain as

$$
\bar{D}_{T_{N^{*}}} T_{N^{*}}=-\frac{\tau}{\kappa} B
$$

Normalizing

$$
\gamma_{N^{*}}=\frac{\tau}{\kappa}
$$

If the curve $\left(\overline{N^{*}}\right)$ is an integral curve of the geodesic spray then $\bar{D}_{T_{N^{*}}} T_{N^{*}}=$ 0 . From here, we get $\tau=0$ and $\kappa=0$. So, we can give the following corollary.

Corollary 2.10 If curve $\alpha^{*}$ is evolute of a planary curve $\alpha$, then the natural lift $\left(\overline{N^{*}}\right)$ of the principal normals indicatrix $\left(N^{*}\right)$ is an integral curves of the geodesic spray on tangent bundle $T\left(S^{2}\right)$ (see example 1$)$.

For the geodesic curvature $\gamma_{B^{*}}$ of the binormals indicatrix curve $\left(B^{*}\right)$ of the evolute curve $\alpha^{*}$ we can write

$$
\begin{gathered}
\gamma_{B^{*}}=\left\|\bar{D}_{T_{B^{*}}} T_{B^{*}}\right\| . \\
\bar{D}_{T_{B^{*}}} T_{B^{*}}=\left(\frac{\cos ^{2}(\varphi+c)}{\sin (\varphi+c)}\right) N+\cos (\varphi+c) B
\end{gathered}
$$

Normalizing

$$
\gamma_{B^{*}}=\cot (\varphi+c)
$$

If the curve $\left(\overline{B^{*}}\right)$ is an integral curve of the geodesic spray then $\bar{D}_{T_{B^{*}}} T_{B^{*}}=$ 0 . Thus $\cos (\varphi+c)=0$. So, we can give the following corollary.

Corollary 2.11 If the tangent vector of evolute curve $\alpha^{*}$ is equal binormal vector of curve $\alpha$, the natural lift $\left(\overline{B^{*}}\right)$ of the binormals indicatrix $\left(B^{*}\right)$ is an integral curves of the geodesic spray on tangent bundle $T\left(S^{2}\right)$.

For the geodesic curvature $\gamma_{C^{*}}$ of the fixed pole curve $\left(C^{*}\right)$ of the evolute curve $\alpha^{*}$ with respect to $S^{2}$ we can write

$$
\gamma_{C^{*}}=\left\|\bar{D}_{T_{C^{*}}} T_{C^{*}}\right\|
$$

By direct calculation, the vector $\bar{D}_{T_{C^{*}}} T_{C^{*}}$ is obtain as

$$
\bar{D}_{T_{C^{*}}} T_{C^{*}}=\frac{\kappa}{\tau} T
$$

Normalizing

$$
\gamma_{C^{*}}=\frac{\kappa}{\tau}
$$

If the curve $\left(\overline{C^{*}}\right)$ is an integral curve of the geodesic spray then $\bar{D}_{T_{C^{*}}} T_{C^{*}}=$ 0 . From here, we get $\kappa=0$ and $\tau \neq 0$. So, we can give the following corollary. 
Corollary 2.12 There is no a evolute of curve $\alpha^{*}$ is such that the fixed pole $\left(C^{*}\right)$ is a great circle on the unit sphere $S^{2}$. Hence the natural lift $\left(\overline{C^{*}}\right)$ of the fixed pole $\left(C^{*}\right)$ is never an integral curves of the geodesic spray on tangent bundle $T\left(S^{2}\right)$.

Corollary 2.13 Let $\alpha^{*}$ be evolute of curve $\alpha$ in $E^{3}$ and $\left\{T^{*}, N^{*}, B^{*}\right\}$ be Frenet-frame of the evolute curve $\alpha^{*}$ at $\alpha^{*}(s)$. For the geodesic curvatures of the spherical indicatrix curves $\left(T^{*}\right),\left(N^{*}\right)$ and $\left(B^{*}\right)$ with the fixed pole curve $\left(C^{*}\right)$ with the respect to $S^{2}$ we have
1) $\gamma_{T^{*}}=\tan (\varphi+c)$
2) $\gamma_{N^{*}}=\frac{\tau}{\kappa}$
3) $\gamma_{B^{*}}=\cot (\varphi+c)$
4) $\gamma_{C^{*}}=\frac{\kappa}{\tau}$

Example 2.14 The evolute of a planary curve $\alpha: I \rightarrow E^{2}, \alpha(t)=\left(t^{2}, t\right)$ is $\alpha^{*}: I \rightarrow E^{2}, \alpha^{*}(t)=\left(\frac{1}{2}\left(1+6 t^{2}\right),-4 t^{3}\right)$ semi-cubic parabola. The principal normals indicatrix of the curve $\alpha^{*}$ is $N^{*}(t)=-\frac{(2 t, 1)}{\sqrt{1+4 t^{2}}}$ and the natural lift $\overline{N^{*}}(t)=\frac{(-2,4 t)}{\left(4 t^{2}+1\right)^{\frac{3}{2}}}$. Then the natural lift $\left(\overline{N^{*}}\right)$ of the principal normals indicatrix $\left(N^{*}\right)$ is an integral curves of the geodesic spray 


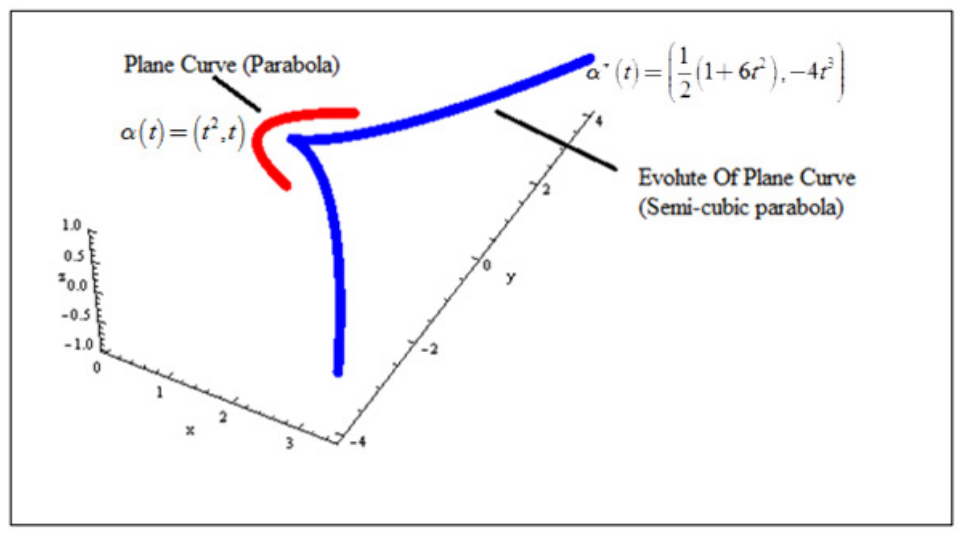

Figure 1: Evolute of Plane Curve

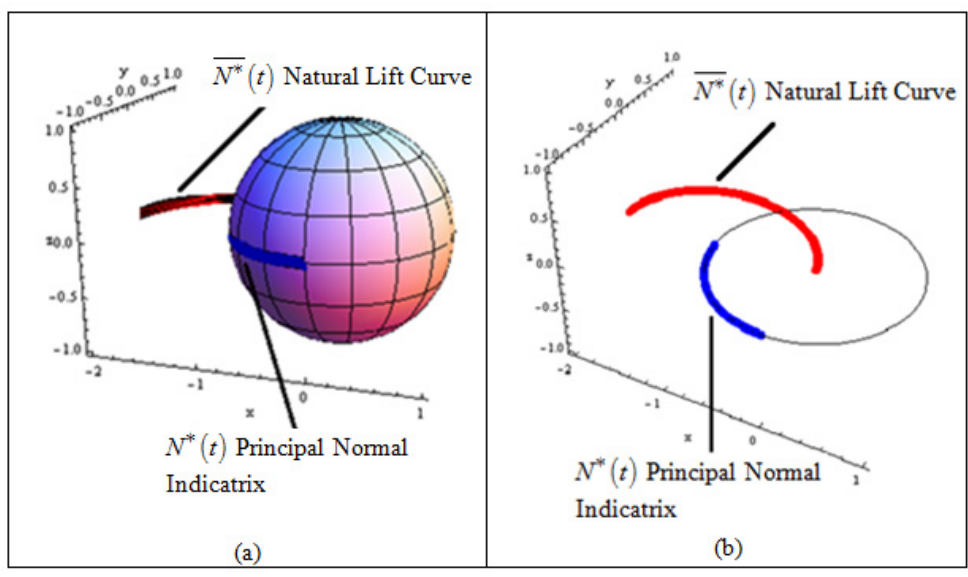

Figure 2: (a), (b): Principal Normal Indicatrix and Its Natural Lift

\section{References}

[1] Çalışkan, M., Sivridağ, A.İ., Hacısalihoğlu, H. H. (1984), Some Characterizations for the Natural Lift Curves and the Geodesic Sprays, Ankara Univ., Faculty of Science, Communications 33, 235-242.

[2] Sivridağ, A.İ., Çalışkan, M. (1991), On the M-Integral Curves and MGeodesic Sprays, Erciyes Univ., Journal of Science (Fen Bilimleri Dergisi), 7(1-2), 1283-1287. 
[3] Sabuncuoğlu, A. , Differential Geometry (in Turkish), Nobel Publishing (2006), Ankara, Turkey.

[4] Hacısalihoğlu, H.H., Differential Geometry (in Turkish), Ertem Publishing (1995),Ankara, Turkey.

[5] Bilici, M., Çalışkan, M. and Aydemir İ. (2002), The Natural Lift Curves and the Geodezic Sprays for the Spherical Indicatrices of the Pair of EvoluteInvolute Curves, International Journal of App. Maths.11,415-420.

[6] Şenatalar, M., Differential Geometry (in Turkish), Istanbul State of Academy of Engineering and Architecture Publishing, 151, Istanbul.

Received: April 27, 2014 